
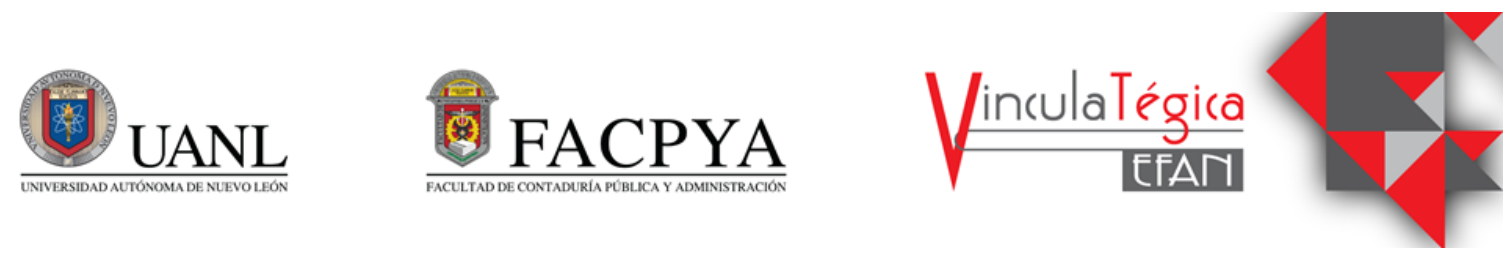

\title{
Determinación de factores que inciden en la decisión de emprender un micronegocio en México
}

\author{
David Orlando García Avilés ${ }^{1}$ \\ ${ }^{1}$ Universidad Autónoma de Nuevo León, david.garciaavl@uanl.edu.mx, Pedro de Alba S/N, Niños Héroes, \\ Ciudad Universitaria, San Nicolás de los Garza, N.L., +52 8183294000
}

Información del artículo revisado por pares

Fecha de aceptación: junio-2021

Fecha de publicación en línea: diciembre-2021

DOI: https://doi.org/10.29105/vtga7.1-109

\begin{abstract}
Resumen
Podemos reconocer que la creación y la continuidad de las microempresas mexicanas son fundamentales para el crecimiento económico del país. Por tanto, el objetivo principal de esta investigación es determinar los motivantes fundamentales que influyen directamente en el emprendimiento de un micronegocio en México. Con base en la hipótesis, existe una importante correlación y repercusión positiva entre el emprendimiento, continuidad y su financiamiento de los micronegocios en México. Para el hallazgo de la solución en base a la pregunta de investigación, se analizarán los datos de las estadísticas nacionales difundidas $\mathrm{y}$ suministradas por el INEGI en la Encuesta Nacional de Micronegocios (ENAMIN) del año 2012. En el transcurso del estudio, se realiza un extenso análisis de cuatro capítulos que consisten en la investigación del tema y finalizando con las conclusiones. Los resultados derivados de esta investigación demuestran de forma clara y precisa, que los aspectos sociodemográficos son los principales factores que tienen una mayor influencia en el emprendimiento de micronegocios en México. El análisis y estudio de los datos presentados por la ENAMIN 2012 son de suma importancia porque brindan una perspectiva del panorama
\end{abstract}

Abstract
We can recognize that the creation and continuity of Mexican microenterprises are fundamental for the economic growth of the country. Therefore, the main objective of this research is to determine the fundamental factors that directly influence microbusiness entrepreneurship in Mexico. Based on the hypothesis, there is an important correlation and positive impact between entrepreneurship, continuity and financing of microbusinesses in Mexico. In order to find the solution based on the research question, will be analyzed data from national statistics published and provided by INEGI in the 2012 National Survey of Microbusinesses (ENAMIN). In the course of the study, an extensive analysis is made in four chapters consisting of an investigation of the subject and ending with the conclusions. The results derived from this research clearly and precisely demonstrate that sociodemographic aspects are the main factors that have the greatest influence on microbusiness entrepreneurship in Mexico. The analysis and examination of the data presented by the ENAMIN 2012 are of utmost importance because they provide a perspective view in which microenterprises in Mexico find themselves at the time of starting their operations. 
en el cual se encuentran las microempresas en México al momento de iniciar sus operaciones.

Palabras clave: micronegocios, emprendimiento, financiamiento de micronegocios.

\section{INTRODUCCIÓN}

Desde hace décadas el estudio de los factores que influyen en la decisión de emprender ha despertado el interés entre los investigadores. Se debe tomar en cuenta que este interés se ve motivado por toda la conmoción que generan dichos factores en los aspectos económicos y sociales a nivel mundial, continental, nacional y local. En los últimos años estos motivantes han estado relacionados o en ocasiones se han utilizado como sinónimo de emprendimiento.

Estos factores de estudio que día con día motivan a todos los empresarios, principalmente de países en vías de desarrollo, en la generación y creación de micro, pequeñas y medianas empresas (MIPyMES), cada vez adquieren mayor relevancia en el mercado, ya que si se busca implementar los programas, apoyos y regulaciones necesarias para que las MIPyMES se adapten en un ambiente adecuado, estas fomentarán, más allá de empleo, innovación y competitividad, un adecuado bienestar social en todos los niveles (Audretsch \& Fritsch, 2002; Fölster, 2000; Hopenhayn, 1992; Klepper, 1996 y Lambson, 1991).

Para que las MIPyMES propicien un crecimiento económico en el país, es de suma importancia que el gobierno central intervenga y formule las políticas públicas más benéficas para este tipo de negocios, enfocándose justamente en las actividades emprendedoras (Reynolds et al., 2002). Uno de los primordiales factores que motivan a todos los empresarios a transformarse de una
Keywords: microbusiness, entrepreneur, microbusiness financing JEL: E24, E26, L26, P45. manera radical, es la globalización. En caso de que estos negocios se muestren renuentes a avanzar junto con el paso que día a día la globalización va imponiendo, pueden caer en el error de alcanzar solamente una pequeña parte de los beneficios económicos de su mercado o hasta en el caso extremo en el cual deban de cesar operaciones.

Para que las MIPyMES propicien un crecimiento económico en el país, es de suma importancia que el gobierno central intervenga y formule las políticas públicas más benéficas para este tipo de negocios, enfocándose justamente en las actividades emprendedoras (Reynolds et al., 2002). Uno de los primordiales factores que motivan a todos los empresarios a transformarse de una manera radical, es la globalización. En caso de que estos negocios se muestren renuentes a avanzar junto con el paso que día a día la globalización va imponiendo, pueden caer en el error de alcanzar solamente una pequeña parte de los beneficios económicos de su mercado o hasta en el caso extremo en el cual deban de cesar operaciones.

Asimismo, Alvarado, Dávila y Vázquez (2018a), en la investigación realizada que lleva por título "Factores que influyen en la continuidad y sobrevivencia de un micronegocio en México", concluyen que el comportamiento de este modelo de negocios está altamente correlacionado con el patrón de características sociodemográficas. Al mismo tiempo, los autores exponen que las variables económicas y del mercado influyen 
más en la determinación de la productividad y rentabilidad de los micronegocios.

Se analizarán los datos de las estadísticas nacionales difundidas y suministradas por el INEGI en la Encuesta Nacional de Micronegocios (ENAMIN) del año 2012. En tanto, la unidad de estudio que se tomó en cuenta, así como también la muestra del mismo, es conformada por todos aquellos propietarios de microempresas familiares y mixtas que integran los diversos sectores económicos y se encuentran establecidas a lo largo de las 32 entidades federativas de la República Mexicana. Por otro lado, la investigación expuesta es de suma importancia ya que revela los efectos que influyen en las variables dependientes sobre la determinación de factores que inciden en la decisión de emprender un micronegocio en México.

\section{MARCO TEÓRICO}

En este capítulo se examina la literatura y se sustentan las variables independientes y dependientes. A su vez, se constituye un análisis documental que incluye la información suficiente que garantiza la investigación presentada en relación con el fenómeno de emprendimiento.

\subsection{Origen y evolución del emprendimiento}

Existen varios conceptos o explicaciones para mostrar lo que realmente significa el emprendimiento. Basado en el estudio y lectura de diversas fuentes, se hablaría del emprendimiento como la actividad de crear un proyecto que brinde soluciones a necesidades del momento o bien mejorar algún proyecto o actividad ya existente.

Al hablar del surgimiento $\mathrm{u}$ origen del emprendimiento estaremos hablando del origen de la humanidad, puesto que el ser humano es creativo por naturaleza y desde sus inicios comenzó a buscar soluciones que le facilitaran su existencia y aseguraran la supervivencia en diversos ambientes. En la historia, el emprendimiento o bien ser emprendedor, hacía referencia a una persona valiente y dispuesta a salir a la aventura, tal fue el caso de Cristóbal Colón al viajar hacia el Nuevo Mundo sin tener certeza de lo que iba a encontrar en dicho lugar (Formichella, 2004).

Fue en el siglo XVI cuando se da el nombre de emprendedores a aquellos soldados y militares que dirigían expediciones, eran proactivos, valientes $\mathrm{y}$ enfocados a la conquista. Urbano y Toledano (2008) mencionan que el término "emprendimiento" surgió en el siglo XVIII en la literatura francesa, y fue el economista francés Richard Cantillon quien lo introdujo, de hecho, el vocablo inicial fue empresario, que significa "entrecoger" o "ir entre".

El economista Jean-Baptiste Say, es uno de los pioneros en exponer el concepto del emprendedor o empresario. Poniendo en contexto la definición de emprendedor o empresario como aquella persona que se desempeña dentro de una empresa. De acuerdo con Jean-Baptiste Say, el emprendedor o empresario lleva consigo una función más importante que la contribución de capital, siendo que lo define como el actor principal que acopla diversos factores productivos, asimismo ejecuta funciones específicas que tienen alto grado de relación con el mero bienestar económico de las mismas empresas.

El emprendimiento es y ha sido una pieza clave en el desarrollo económico e industrial a lo largo de la historia. Barreto (1989) expone que en 1880 Alfred Marshall fue quien reconoció por primera vez, de manera formal, lo necesario que era para la producción el vincular el emprendimiento o entrepreneurship. Sin duda, la relación que existe entre la economía y el emprendimiento ha ido evolucionando junto con las nuevas corrientes, y también se va desarrollando a través del tiempo.

Fue a finales del siglo XIX y principios del XX que la corriente $y$ el nuevo pensamiento económico neoclásico coincidieron de una manera mayormente fluida con el emprendimiento, puesto que el objetivo era acrecentar y mejorar las grandes industrias y la libertad del comercio.

Basado en los hechos históricos, se podría hablar del siglo XX como la época de 
transición hacia el reconocimiento del emprendimiento como parte de la actividad económica de un país o del mundo entero. El economista austro-estadounidense Joseph Alois Schumpeter habló de lo importante que es la función directiva en un país (Gil y Celma, 2002).

Para Schumpeter, los empresarios son personas que dirigen la realización de nuevas combinaciones con apertura a nuevas posibilidades, tales como:

- Introducción de un nuevo bien.

- Introducción de un nuevo proceso de producción.

- Abrir un nuevo mercado.

- Crear una nueva organización dentro de una industria.

Así también, una figura importante en la historia del concepto de emprendimiento y el empresario es Frank Knight, un economista estadounidense. Tarapuez, Zapata y Agreda (2008) mencionan que el principal aporte que Knight hizo fue la distinción entre la incertidumbre y el riesgo del emprendimiento.

El siglo XXI ha traído diversos cambios en los diversos sistemas establecidos, estamos hablando de temas desde lo político, social, cultural y, por supuesto, económico. Una mayor apertura en los mercados, el fácil acceso a las redes de comunicación, el avance en la tecnología y el desarrollo de aplicaciones se deben básicamente a un grupo de personas que han tenido la visión de comenzar un proyecto y desarrollarlo, de modo que invierten tanto dinero como esfuerzo y estudio para consolidar dicho plan y/o proyecto.

Israel Meir Kirzner es un economista austriaco muy reconocido. En 2006 ganó el Premio Mundial de Investigación en Emprendimiento; en dicha investigación remarca la función vital de un emprendedor/empresario para el crecimiento económico (Mi Propio Jefe, 2020).

García y Bòria, (2006) mencionan que el mercado presenta oportunidades de negocio de manera constante, debido a diversos factores como lo son los cambios sociales y el entorno dinámico y variable del mercado. Esto de alguna manera hace que el mercado demande un alto grado de satisfacción.

Puesto que el emprendimiento siempre ha existido en nuestra naturaleza humana buscando la forma de salir a flote, el emprendedor se ha convertido en un sujeto propio de la sociedad neoliberal. El emprendimiento es una categoría social, una forma de vida, un modelo cultural, un modo de estar y de relacionarse con el mundo que se ha profesionalizado (Ateljevic \& Page, 2009). De cierta forma, podría decirse que el emprendimiento es un factor de cambio y un camino para llegar a satisfacer desde una necesidad básica hasta un capricho o lujo.

De lo anterior, se concluye que el emprendimiento siempre ha existido y conforme avanza la humanidad y el desarrollo es mayor y existen mejoras, el emprendimiento seguirá avanzando de la misma forma ya que es una acción inherente al ser humano y sus actividades económicas y sociales.

\subsection{Concepto de emprendimiento}

A lo largo de la historia se han definido los conceptos de emprendimiento y emprendedor; diversos autores, pensadores y especialistas en materia de economía han compilado una serie de factores y herramientas para concluir en dar vida a un concepto acertado de la palabra emprendimiento. En algunos casos, se ha visto la palabra emprendimiento como sinónimo de empresario, sin embargo, cabe mencionar que el significado otorgado a ambos conceptos puede variar en gran medida, creando una confusión si se toman como sinónimos.

Existe una gran diferencia entre el concepto de emprendedor y empresario. El emprendedor se puede definir como la persona que genera resoluciones y actúa ante situaciones de alta dificulta. En cambio, un empresario es aquel personaje que es el directivo o titular de una empresa o negocio (Bucardo et al., 2015). En seguida en la Tabla 1 se muestra una serie de conceptos de autores diversos sobre el emprendimiento

Tabla 1. Definiciones del concepto de emprendedor.

Autor 


\begin{tabular}{|c|c|}
\hline $\begin{array}{l}\text { Richard Cantillon } \\
\text { (1975) }\end{array}$ & $\begin{array}{l}\text { Una persona que se } \\
\text { caracteriza por } \\
\text { productos a precios } \\
\text { conocidos para venderlos } \\
\text { a precios desconocidos. }\end{array}$ \\
\hline $\begin{array}{r}\text { Schu } \\
(1)\end{array}$ & $\begin{array}{l}\text { Ente generador de } \\
\text { crecimiento económico. } \\
\text { Destructor } \\
\text { (proceso de desarrollo } \\
\text { caracterizado por la } \\
\text { innovación constante). }\end{array}$ \\
\hline $\begin{array}{l}\text { Cantillon, Thunen } \\
\text { y Baudeau } \\
\text { (citados en } \\
\text { Rodríguez, 2009) }\end{array}$ & $\begin{array}{lrr}\text { Tomador de } & \text { riesgo y } \\
\text { persona } & \text { demasiado } \\
\text { inteligente. } & \end{array}$ \\
\hline $\begin{array}{r}\text { Benthar } \\
\quad \text { (cita } \\
\text { Rodrígu }\end{array}$ & $\begin{array}{l}\text { Tomador de decisiones } \\
\text { con riesgo innovador y } \\
\text { como } \\
\text { innovador. }\end{array}$ \\
\hline Pereira (2003) & $\begin{array}{llll}\text { Es quien } & \text { percibe } & \text { la } \\
\text { oportunidad } & \text { y } & \text { crea } & \text { la } \\
\text { organización } & & & \text { para } \\
\text { aprovecharla. } & & & \end{array}$ \\
\hline De Vrie & $\begin{array}{l}\text { Concibe e implementa } \\
\text { una idea a través de la } \\
\text { innovación, } \\
\text { administración y toma de } \\
\text { riesgos. }\end{array}$ \\
\hline Dru & $\begin{array}{l}\text { Ejecuta las tareas de } \\
\text { proyección, combinación, } \\
\text { innovación y anticipación. }\end{array}$ \\
\hline Baun & $\begin{array}{l}\text { Es más que un } \\
\text { organizador y que un } \\
\text { innovador, debe conseguir } \\
\text { nuevas fuentes de } \\
\text { recursos y métodos de } \\
\text { mercadeo y estructurar } \\
\text { una nueva organización. }\end{array}$ \\
\hline Dees (1998) & $\begin{array}{l}\text { Es capaz de destrucción } \\
\text { creativa, creación de } \\
\text { valor, identificación de } \\
\text { oportunidades e ingenio. }\end{array}$ \\
\hline
\end{tabular}

Fuente: Bucardo, Saavedra y Camarena (2015).

El concepto de emprendimiento puede tener diversas variaciones, como lo podemos ver en la Tabla 1. El concepto puede parecer cambiar de autor a autor o, a su vez, de época en época. Sin embargo, se coincide en que la persona emprendedora debe poseer ciertas características, tales como:

- Ser una persona dispuesta a aceptar y afrontar nuevos retos,

- Innovar y crear soluciones y

- Buscar nuevas oportunidades y tomar decisiones.

Uribe y Reinoso (2013) definen el concepto de emprendimiento además como la función del individuo para generar desde significados y aprender a resolver inconvenientes basado en sus emociones, su creatividad, sus ocupaciones y valores individuales, lo cual le posibilita acoplarse a un entorno y aprovechar las oportunidades que este ofrece para beneficio propio y de la sociedad.

El término puede tender a tomar diversas maneras; generalmente, se plantea que un espíritu emprendedor es el comportamiento de una persona que visualiza oportunidades, organiza recursos y recibe resultados de diversa índole: ya sean económicos, sociales, cooperativos, públicos, entre otros (Leiva, 2013).

Cuando se trata de explicar el emprendimiento en todas sus facetas se deben tomar en cuenta factores específicos como lo son el nivel socioeconómico del país donde se está desarrollando el proyecto hasta las razones por las que se está generando dicho emprendimiento, tal como se mencionaba en párrafos anteriores.

Basado en los datos anteriores concluimos que el emprendimiento es una actividad en la que aceptar y afrontar nuevos retos y buscar siempre avance e innovación son puntos clave; sin embargo, el concepto se expande al momento de hablar de los diferentes mercados, puesto que factores como el nivel socioeconómico, educación y propósito dan paso a una definición específica de cada forma de emprendimiento.

\subsection{Tipos de emprendimiento}

Actualmente, nos encontramos en una era de evolución y reajuste en los ideales y formas de organización tanto en lo político como en lo económico, social y cultural. Estamos viviendo la adaptación y aceptación hacia una forma de vida amplia y abierta en la que se pretende aceptar al otro sin importar las diferencias y con este crecimiento continuo los mercados se vuelven cada vez más exigentes.

Lo anterior deja una puerta de oportunidad para nuevos proyectos de 
emprendimiento que puedan cumplir con las expectativas tanto en productos como en servicios, lo que también ha obligado a las grandes empresas y corporativos a acondicionar sus formas hacia las nuevas exigencias, con el riesgo de errar.

El término emprendimiento podría ser asociado a una vasta gama de negocios que, no obstante, experimentan fuertes diferencias en su fondo y en su forma.

El emprendimiento tiene distintas aproximaciones; además, cuenta como un componente de aumento de las economías y principalmente se aborda a partir de la perspectiva de construcción de organizaciones (Zárate et al., 2013).

Enseguida se muestran los principales tipos de emprendimiento y su respectiva exposición, según el GEM (2018):

- Emprendimiento por oportunidad: Es la actividad que se inicia para explotar alguna posibilidad de comercio.

- Emprendimiento por necesidad: Nace una vez que no hay otras alternativas de trabajo o las existentes son insatisfactorias (Reynolds, 2018).

- Emprendimiento innovador: A nivel macroeconómico, el emprendimiento basado en la innovación se considera un factor que impulsa el desarrollo económico (Urbano et al., 2019b). Existe una estrecha relación entre emprendimiento, innovación y desarrollo económico. El mayor ejemplo es "la distinción entre países avanzados y en desarrollo". La innovación en los negocios y las herramientas con las que se cuentan pueden generar mejoras considerables en la economía de un país o región.

- Emprendimiento local y sistemático: Tiene sus raíces en el carácter informal de los mercados y se limita el alcance de la idea empresarial proyectándose sólo a grado local. Esta clase de emprendimiento es dependiente de las oportunidades que da el mercado (Sautet, 2013).

- Emprendimiento formal e informal: $\mathrm{El}$ emprendedor informal es ese que participa activamente en la administración de una totalmente nueva organización dedicada a la comercialización de bienes y servicios legítimos y no se ha registrado frente a las autoridades oficiales (Autio $\& \mathrm{Fu}, 2014)$. Sin embargo, los autores Dau y Cuervo-Cazurra (2014) aseguran que la idea empresarial formal corresponde a la construcción de novedosas organizaciones que permanecen registradas legalmente en un territorio definido.

Basado en el estudio de los datos que arroja el GEM (2020) se concluye que los diferentes tipos de emprendimiento que existen alrededor del mundo tienen toda relación con el nivel del país y el estándar de la sociedad. Diversos factores como lo son la edad de la población, la cultura, el sistema educativo y el apoyo que reciben los emprendedores por parte del gobierno juegan un papel importante al momento de decidir cuál será el tipo de emprendimiento a tomar y actuar en el mismo.

\subsection{Los micronegocios en México}

México se caracteriza por ser un país ingenioso. Ante la mirada de los demás países, se tiene una imagen que muestra cómo el mexicano siempre busca la ocasión para implementar su imaginación y poner en práctica la forma creativa de su persona, improvisando y generando soluciones rápidas.

Al tratarse México de un país donde la tasa de desempleo equivale al 5\% de la Población Económicamente Activa (PEA), 
sus habitantes se encuentran en una posición en la que seguir buscando una oportunidad laboral, en muchas ocasiones no es su única opción.

Tal es la importancia de los micronegocios en el país que en el año 2012 y años anteriores, el INEGI diseñó y generó la ENAMIN con el principal propósito de llevar a cabo un estudio exhaustivo sobre las actividades de este sector, así como de sus impactos sociales, económicos y financieros.

Según la Encuesta Nacional sobre Productividad y Competitividad de las micro, pequeñas y medianas empresas (ENAPROCE), podemos analizar, con apoyo, que en el año 2018 en el país existía un total de 4 millones 57 mil 719 microempresas, con una participación en el mercado equivalente al 97.3\% (INEGI, 2018).

A lo largo de la historia, la acción de emprender o el concepto de emprendimiento en sí han tenido diferentes definiciones, pero todas se resumen en crear y llevar una idea a los hechos por muy disparatado o fuera de lo común que dicho proyecto parezca.

El impacto económico que la microempresa tiene en el país es básico y es considerado una parte fundamental del PIB dado que, según datos del INEGI (2018), le aporta un $52 \%$. Pareciera que, debido a la situación mundial actual, este panorama seguirá en aumento pues muchos de los grandes corporativos pusieron en pausa todas aquellas contrataciones, proyectos $\mathrm{O}$ aperturas.

A lo largo de los años, múltiples estudios y autores han tratado de explicar el fenómeno del emprendimiento de micronegocios en México. Es interesante conocer que muchos de dichos negocios no comenzaron como una primera opción para sus dueños; muchos parecen ser opciones temporales mientras estos encontraban un trabajo, o facilitadas por la conveniencia (Peña et al., 2012).

Según los censos económicos de 2019, en México había 6.3 millones de establecimientos con 36 millones de personas ocupadas en ellos; de estos, el $94.9 \%$ era microempresa, $4.9 \%$ era PYME y apenas el $0.2 \%$ era grande empresa (García, 2020).
Los micronegocios en México cumplen un papel elemental para la integración social, conforman un eslabón determinante en la cadena de la actividad económica y son fundamentales para el desarrollo regional y local.

Es indispensable que autoridades conducentes instrumenten ocupaciones que contribuyan de forma decisiva en el desarrollo y consolidación de estos negocios debido a que son pieza importante de la economía nacional, como declaró el senador Juan Zepeda en un comunicado social (Senado de la República, 2020).

\section{MÉTODO}

Este apartado está conformado por la metodología y el diseño de la investigación implementada para la obtención de los resultados acerca de las hipótesis establecidas y así poder llegar al análisis de la determinación de factores que inciden en la decisión de emprender un micronegocio en México, así como de los principales motivantes que influyen en el momento del emprendimiento. Para alcanzar nuestro objetivo principal, se toman en cuenta los resultados arrojados de las preguntas número 80 a la 90 de la ENAMIN 2012.

La investigación es de tipo cuantitativa y correlacional. Como se mencionó anteriormente, en este trabajo se busca conocer el grado de las variaciones de los factores, en relación con otros factores, para identificar la relación de la determinación de aquellos que inciden en la decisión de emprender un micronegocio.

El aspecto cuantitativo del análisis nos ayudará a conocer la verdad de una forma justa ya que se recogen y analizan los datos por medio de los conceptos y las cambiantes estudiadas usando datos estadísticos nacionales recolectados a través de instrumento estructurado, y analizándolos con rigor para contrastar las premisas propuestas en la indagación.

De igual manera, la investigación es del tipo correlacional debido a que se evalúan las interacciones que tienen la posibilidad de existir entre la variable dependiente y las independientes que se 
hallan sujetas adentro de la misma investigación (Hernández et al., 2016).

Durante el desarrollo de la investigación, se realiza el planteamiento de la hipótesis general. En la cual se redacta que el financiamiento de los micronegocios y los primordiales motivos para el emprendimiento de estas unidades de negocio en Mexico, se hallan estrechamente asociadas e impactan de manera positiva su continuidad.

La información estadística nacional presentada por el INEGI a través de la Encuesta Nacional de Micronegocios (ENAMIN) del año 2012, en la presente investigación se lleva a cabo un análisis exhaustivo de los sectores económicos que van desde la manufactura, comercio, construcción y de servicios a lo largo de toda la República Mexicana.

El primordial objetivo, de esta herramienta de recolección de datos diseñado y empleado por el INEGI, ha sido para recabar información de las microempresas en las cuales su capacidad gremial sea a partir de una a seis personas (incluyendo al dueño) y hasta 16 si hablamos de microempresas en el giro manufacturero. La encuesta organizada, administrada y personal a grado nacional, ha sido la técnica aplicada en la ENAMIN.

De 71 a 100 preguntas son las variaciones que tenemos la posibilidad de hallar en los formularios, según sea la situación del año de su aplicación. Como lo hemos dicho antes, la investigación presente examina a grado nacional los resultados que corresponden al año 2012. Las secciones e interrogantes de la ENAMIN que fueron usadas son solo esas pertenecientes al eje temático de financiamiento.

De acuerdo con el instrumento utilizado en la ENAMIN del año 2012, en el cuestionario se desglosan las variables, señaladas en la siguiente sección, a partir de la pregunta número 80 a la 90 .

\section{RESULTADOS}

En este capítulo se muestran los resultados de las herramientas definidas en el capítulo anterior. Asimismo, se presentan los estadísticos descriptivos de la base de datos. Analizando los resultados conseguidos de los propietarios de microempresas familiares, se demuestra que, en esta clase de organizaciones a grado nacional, hay una justa distribución de género entre los propietarios masculinos y femeninos de dichos micronegocios.

Los datos encontrados en relación con los micronegocios familiares a nivel nacional, se localizó que casi la mitad de esta clase de negocios son dirigidos por propietarios más grandes de 41 años edad (Tabla 2). El segundo segmento más enorme en este tipo de negocios, lo componen los propietarios que se hallan en el rango de 29 a 40 años de edad, y se tiene un porcentaje de $10.13 \%$ de propietarios adolescentes en el rango de los 18 a 28 años de edad.

Tabla 2. Resultados descriptivos de edad.

\begin{tabular}{ccc}
\hline \multicolumn{3}{c}{ Edad de las personas entrevistadas } \\
\hline \multicolumn{3}{c}{ Menos de 18} \\
años & Frecuencia & Porcentaje \\
$\begin{array}{c}\text { De 18 a } 28 \\
\text { años }\end{array}$ & 2,531 & $0.38 \%$ \\
$\begin{array}{c}\text { De 29 a } 40 \\
\text { años }\end{array}$ & 6,913 & $10.13 \%$ \\
$\begin{array}{c}\text { De 41 a } 52 \\
\text { años }\end{array}$ & 7,943 & $31.80 \%$ \\
$\begin{array}{c}\text { De 53 a } 64 \\
\text { años }\end{array}$ & 5,210 & $20.86 \%$ \\
$\begin{array}{c}\text { De 65 a 76 } \\
\text { años }\end{array}$ & 1,897 & $7.60 \%$ \\
$\begin{array}{c}\text { De 77 a } 88 \\
\text { años }\end{array}$ & 366 & $1.47 \%$ \\
$\begin{array}{c}\text { De 89 a } 98 \\
\text { años }\end{array}$ & 20 & $0.08 \%$ \\
Total & 24,975 & $100 \%$ \\
\hline
\end{tabular}

*Los datos se representan con el factor de expansión determinado por el INEGI (2012).

**El total puede variar debido al factor de expansión de cada pregunta y el número de valores perdidos.

Fuente: elaboración propia con datos de la ENAMIN (2012).

En cuanto a la ocupación laboral en la operación de las microempresas en México de acuerdo con la ENAMIN, se encuentra que la mayoría de estas está constituida por dos personas. Enseguida, se encuentra un porcentaje de $5.90 \%$ en el que opera 
solamente una persona, que, por ende, se trata del dueño del micronegocio (Tabla 3).

Tabla 3. Datos generales del encuestado. ¿Aproximadamente cuántas personas, incluyendo al dueño, laboraban donde usted trabajaba?

\begin{tabular}{|c|c|c|}
\hline & Frecuencia & Porcentaje \\
\hline 1 & 1,471 & $5.90 \%$ \\
\hline 2 & 5,429 & $21.70 \%$ \\
\hline 3 & 1,283 & $5.10 \%$ \\
\hline 4 & 571 & $2.30 \%$ \\
\hline 5 & 503 & $2 \%$ \\
\hline 6 & 469 & $1.90 \%$ \\
\hline 7 & 543 & $2.20 \%$ \\
\hline 8 & 683 & $2.70 \%$ \\
\hline 9 & 596 & $2.40 \%$ \\
\hline 10 & 357 & $1.40 \%$ \\
\hline 11 & 902 & $3.60 \%$ \\
\hline 99 & 204 & $0.80 \%$ \\
\hline Total & 13,011 & $52.10 \%$ \\
\hline $\begin{array}{c}\text { Perdidos } \\
\text { sistema }\end{array}$ & 11,964 & $47.90 \%$ \\
\hline Total & 24,975 & $100 \%$ \\
\hline
\end{tabular}

*Los datos se representan con el factor de expansión determinado por el INEGI (2012).

**El total puede variar debido al factor de expansión de cada pregunta y el número de valores perdidos.

Fuente: elaboración propia con datos de la ENAMIN (2012).

Los resultados del análisis sobre cómo se encuentra estructurada la pertenencia del micronegocio, en la cual podemos encontrar una notable diferencia entre los micronegocios donde una sola persona es propietaria, en comparación con los que se encuentran conformados por varios propietarios.

La Tabla 4 muestra los porcentajes de negocios en función de la persona o personas que iniciaron la operación, según la ENAMIN. En este tipo de modelo de negocio se aprecia que el arranque se da, principalmente, de la siguiente manera: solo(a) (81.20\%), usted y su pareja o cónyuge (u otro familiar) $(8.10 \%)$, otro(s) familiar(es) $(4.30 \%) \mathrm{y}$, por último, con su pareja o cónyuge (4\%).

Tabla 4. Datos generales del encuestado. ¿Quién empezó este negocio o actividad y en qué fecha?

\begin{tabular}{ccc}
\hline & Frecuencia & Porcentaje \\
\hline Usted solo(a) & 20,268 & $81.20 \%$
\end{tabular}

$\begin{aligned} & \text { Su pareja o } \\ & \text { cónyuge }\end{aligned}$
Usted y su pareja o
cónyuge (u otro
familiar)
Usted y otra(s)
persona(s) no
familiares
Otro(s) familiar(es)
Otra(s) persona(s)
Total
$\begin{aligned} & \text { *Los datos se representan con el factor de expansión } \\ & \text { determinado por el INEGI (2012). }\end{aligned}$
**El total puede variar debido al factor de expansión de cada
pregunta y el número de valores perdidos.
Fuente: elaboración propia con datos de la ENAMIN (2012).
$\quad \begin{aligned} & \text { En la Tabla } 5 \text { se describen los } \\ & \text { resultados del análisis correspondiente sobre }\end{aligned}$
los principales motivos por los cuales se
inició el negocio, según la ENAMIN. Se
puede notar que los motivos que con más
frecuencia impulsan la creación, arranque y
desarrollo de los micronegocios en México
son: complementar el ingreso familiar
(25.70\%), mejorar el ingreso (21.70), ejercer
un oficio, carrera o profesión (11.20\%) y fue
la única manera que se tuvo para obtener un
ingreso (11.10\%).

Tabla 5. Datos generales del encuestado. ¿Cuál es el motivo principal por el que se inició este negocio o actividad?

\begin{tabular}{ccc}
\hline & Frecuencia & Porcentaje \\
\hline $\begin{array}{c}\text { Por tradición } \\
\text { familiar o lo } \\
\text { heredó } \\
\quad \text { Para }\end{array}$ & 1,569 & $6.30 \%$ \\
$\begin{array}{c}\text { complementar el } \\
\text { ingreso familiar } \\
\text { Para mejorar el } \\
\text { ingreso }\end{array}$ & 6,424 & $25.70 \%$ \\
$\begin{array}{c}\text { Tenía dinero y } \\
\text { encontró una } \\
\text { buena }\end{array}$ & 1,219 & $21.70 \%$ \\
$\begin{array}{c}\text { oportunidad } \\
\text { Para ejercer su } \\
\text { oficio, carrera o } \\
\text { profesión }\end{array}$ & 2,796 & \\
Fue la única \\
manera que tuvo \\
para obtener un \\
ingreso \\
$\begin{array}{c}\text { No tenía la } \\
\text { experiencia }\end{array}$ & 2,767 & $11.20 \%$ \\
& & \\
\end{tabular}




\begin{tabular}{|c|c|c|}
\hline $\begin{array}{l}\text { requerida para } \\
\text { un empleo }\end{array}$ & & \\
\hline $\begin{array}{l}\text { No tenía la } \\
\text { escolaridad o } \\
\text { capacitación }\end{array}$ & 100 & $0.40 \%$ \\
\hline $\begin{array}{l}\text { requerida para } \\
\text { un empleo }\end{array}$ & & \\
\hline $\begin{array}{c}\text { Estaba } \\
\text { sobrecapacitado } \\
\text { para un empleo }\end{array}$ & 14 & $0.10 \%$ \\
\hline Los empleos que & & \\
\hline $\begin{array}{l}\text { encontró estaban } \\
\text { mal pagados }\end{array}$ & 228 & $0.90 \%$ \\
\hline $\begin{array}{l}\text { Requería un } \\
\text { horario flexible }\end{array}$ & 475 & $1.90 \%$ \\
\hline No había & & \\
\hline $\begin{array}{l}\text { oportunidades de } \\
\text { empleo }\end{array}$ & 1,211 & $4.80 \%$ \\
\hline Otra razón & 2,712 & $10.90 \%$ \\
\hline Total & 24,975 & $100 \%$ \\
\hline
\end{tabular}

En cuanto a los trámites ante alguna dependencia de gobierno para poder iniciar el negocio o actividad, nos podemos percatar que, hasta cierto punto, existe informalidad, ya que el $71.70 \%$ de los micronegocios en México inicia sus operaciones sin antes haber realizado los trámites pertinentes para comenzar a operar de manera formal.

\section{CONCLUSIONES}

¿Cuáles son los factores que inciden en la decisión de emprender un micronegocio en México?, esta es la pregunta de investigación que se planteó al comienzo de esta investigación. La cual nos ayudó a encontrar de forma clara y precisa, que los aspectos sociodemográficos son los principales factores que tienen una mayor influencia en el emprendimiento de micronegocios en México.

Esta tesina condujo a un análisis cuantitativo y correlacional basado en la ENAMIN 2012. Al respecto, se encontró que el inicio de las operaciones de los micronegocios está influenciado principalmente por dos tipos de asociaciones. Ya sea que estos arranquen operando de manera individual o por una sola persona (propietario-emprendedor) con un porcentaje del $81.20 \%$ y con el $8.10 \%$ por una pareja u otro familiar. Los resultados revelan que las principales razones para empezar o emprender un micronegocio son para completar $(25.70 \%)$ y mejorar el ingreso familiar $(21.70 \%)$.

$\mathrm{Al}$ momento en que estos micronegocios comienzan a operar, se encuentra que la gran mayoría no realiza trámites ante alguna dependencia de gobierno $(71.10 \%)$, lo cual abre una gran brecha entre los que se encuentran operando de manera informal y los que se encuentran operando de manera formal.

La razón principal por la cual los microempresarios se acercan a una dependencia de gobierno para hacer trámites es para registrar o dar de alta el negocio o actividad que van a desempeñar (9.4\%); por otros motivos, se encuentra el $2 \%$ y para dar de alta o baja a trabajadores, acude a estas dependencias el $0.20 \%$.

El análisis y estudio de los datos anteriormente presentados son de suma importancia porque brindan una perspectiva del panorama en el cual se encuentran las microempresas en México al momento de iniciar sus operaciones. Al mismo tiempo, podemos encontrar todas las razones sociodemográficas en las cuales los micronegocios se ven envueltos.

Los resultados de esta investigación son consistentes con lo que Alvarado et al. (2018b) indican en el sentido de que el comportamiento de las microempresas está altamente correlacionado con el patrón de características sociodemográficas. Peña et al. (2012) hicieron uso de un modelo probabilístico de regresión múltiple en el cual se encontró que las microempresas no son iguales, ya que las razones para iniciarlos varían de acuerdo con las expectativas de los dueños de los micronegocios.

De acuerdo con los resultados obtenidos a lo largo de esta investigación, nos podemos percatar que el objetivo general que se abordó en el comienzo de esta investigación se ha llegado cumplir debido a que se muestra la información, 
datos y referencias suficientes para determinar cuáles son los factores que inciden en la decisión de emprender un micronegocio en México.

$\mathrm{Al}$ instante de hacer este trabajo de investigación, se adquirió el conocimiento suficiente para descubrir varias limitantes y oportunidades de optimización que tienen la posibilidad de ser de gran utilidad para futuras investigaciones fundamentadas en los componentes que inciden en la elección de emprender un micronegocio en México. Ejemplificando conceptualmente, se puede hacer un comparativo de las mismas variables en los diferentes estados o zonas de la República Mexicana. De forma que esto podría mejorar el razonamiento y la percepción que se tiene en la actividad empresarial en relación a esta sociedad de comercio en el territorio nacional.

Así mismo, los resultados han propuesto varias preguntas que exigen el análisis de otras variables que no fueron analizadas en este trabajo y que podrían mejorar el razonamiento. Es viable obtener resultados más certeros si se desarrolla un comparativo por género y se utilizan otras técnicas estadísticas en el estudio de la muestra, tomando en cuenta los diferentes años de levantamiento de la ENAMIN. Con esto se podría tener más información importante para examinar y entender las propiedades primordiales de los micronegocios en México. 


\section{REFERENCIAS}

Alvarado, E. L., Dávila, M. A., y Vázquez, M. Z. (2018a). Factores que influyen en la continuidad y sobrevivencia de un micronegocio en México. Contaduría y Administración, 63(3), 120.

Alvarado, E. L., Ortiz, J. R., y Morales, D. R. (2018b). Factores que influyen en el emprendimiento de un micronegocio familiar en México. Revista de Economía del Rosario, 21(1), 153180.

Ateljevic, J., \& Page, S. (2009). Tourism and Entrepreneurship: International Perspectives. Elsevier.

Audretsch, D. B., \& Fritsch, M. (2002). Growth regimes over time and space. Regional Studies, 36(2), 113-124.

Autio, E., \& Fu, K. (2014). Economic and political institutions and entry into formal and informal entrepreneurship. Asia Pacific Journal of Management, (32), 67-94.

Barreto, H. (1989). The Entrepreneur in Microeconomic Theory. Routledge.

Bucardo, M. A., Saavedra, M. L., y Camarena, M. E. (2015). Hacia una comprensión de los conceptos de emprendedores y empresarios. Suma de Negocios, 6(13), 98-107.

Dau, L. A., \& Cuervo-Cazurra, A. (2014). To formalize or not to formalize: Entrepreneurship and pro-market institutions. Journal of Business Venturing, 29(5), 668-686.

Fölster, S. (2000). Do entrepreneurs create jobs? Small Business Economics, 14(2), 137-148.

Formichella, M. M. (enero de 2004). El concepto de emprendimiento y su relación con la educación, el empleo y el desarrollo local. Ministerio de Asuntos Agrarios y Producción-Pcia. de Buenos Aires.

García, A., y Bòria, S. (2006). Los nuevos emprendedores. Creación de empresas en el siglo XXI. Universitat de Barcelona.

García, M. (17 de julio de 2020). Predominan los micronegocios en México, pero empresas tienen $54.7 \%$ del valor agregado: INEGI. Diario Portal.

Gil, M. D., y Celma, M. D. (2002). Concepto, contenido y objeto de la administración de empresas. Editorial UOC.

Global Entrepreneurship Monitor. (2018). Global Entrepreneurship Monitor. https://www.gemconsortium.org/about/gem/5

Global Entrepreneurship Monitor. (24 de noviembre de 2020). Entrepreneurship Behaviour and Attitudes. Global Entrepreneurship Monitor. https://www.gemconsortium.org/data

Hernández, R., Fernández, C., y Baptista, M. (2016). Métodos de investigación: General. McGrawHill.

Hopenhayn, H. A. (1992). Entry, exit, and firm dynamics in long run equilibrium. Econométrica, 60(5), 1127-1150.

Instituto Nacional de Estadística y Geografía. (2015). Esperanza de vida de los negocios a nivel nacional y por entidad federativa. https://www.inegi.org.mx/temas/evnm/doc/evn_ent_fed.pdf

Instituto Nacional de Estadística y Geografía. (2018). Encuesta Nacional sobre Productividad y Competitividad de las Micro, Pequeñas y Medianas Empresas (ENAPROCE) 2018. https://www.inegi.org.mx/programas/enaproce/2018/

Klepper, S. (1996). Entry, exit, growth, and innovation over the product life cycle. The American Economic Review, 86(3), 562-583.

Lambson, V. E. (1991). Industry evolution with sunk costs and uncertain market conditions. International Journal of Industrial Organization, 9(2), 171-196.

Leiva, J. C. (2013). Los emprendedores y la creación de empresas. Editorial Tecnológica de Costa Rica.

Mi Propio Jefe. (16 de octubre de 2020). El papel del empresario, según Israel M. Kirzner. Recuperado el 26 de noviembre de 2020, de https://mipropiojefe.com/el-papel-delempresario-segun-israel-m-kirzner/

Peña, P., Ríos, I., y Salazar, S. (2012). Los micronegocios en México: Razones para emprenderlos, 
expectativas, tamaño y financiamiento. Estudios Económicos CNBV, 1, 75-94.

Reynolds, P. D. (2018). Business creation: Ten factors for entrepreneurial success. Edward Elgar Publishing Limited.

Reynolds, P. D., Bygrave, W. D., Autio, E., Cox, L. W., \& Hay, M. (2002). Global Entrepreneurship Monitor. Executive Report. Babson College; Ewing Marion Kauffman Foundation; London Business School.

Sautet, F. (2013). Local and systemic entrepreneurship: Solving the puzzle of entrepreneurship and economic development. Entrepreneurship: Theory and Practice, 37(2), 387-402.

Secretaría de Economía. (2010). Microempresas. http://www.20062012.economia.gob.mx/mexicoemprende/empresas/microempresario\#: :text=Las\%20microempresas\%20son\%20todos $\% 20$ aquellos,ciento\%20de1\%20Producto\%20Interno\%20Bruto.

Senado de la República. (29 de febrero de 2020). Pymes, importante motor para el desarrollo económico nacional. En: http://comunicacion.senado.gob.mx/index.php/informacion/boletines/47767-pymesimportante-motor-para-el-desarrollo-economico-nacional-mc.html

Tarapuez, E., Zapata, J., y Agreda, E. (2008). Knight y sus aportes a la teoría del emprendedor. Estudios Gerenciales, 24(106), 83-98.

Urbano, D., Aparicio, S., \& Audretsch, D. B. (2019b). The effect of entrepreneurial activity on economic growth. In Institutions, entrepreneurship, and economic performance (Vol. 41, pp. 85-106), Springer International Publishing.

Urbano, D., y Toledano, N. (2008). Invitación al emprendimiento. Una aproximación a la creación de empresas. Editorial UOC.

Uribe, M. E., y Reinoso, J. F. (2013). Emprendimiento y empresarismo (1 ed.). Ediciones de la U.

Zárate, R., Argüelles, D., Aparicio, S., Salcedo, C., Ibarra, A., Matiz, F., Parra, L., y Argote, M. (2013). Emprendimiento: diferentes aproximaciones. Ediciones EAN. 